\title{
Dynamic Evaluation of Processive Movement by Individual Supermolecular Ferritin using High-Speed AFM
}

\author{
S-I. Yamamoto ${ }^{1)}$, K. Tomizaki ${ }^{2}$, H. Yoshioka ${ }^{3)}$, Y. Uraoka ${ }^{4)}$, I. Yamashita ${ }^{5)}$ and S. Hasegawa ${ }^{6}$ \\ 1) Department of Electronics and Informatics, Faculty of Science and Technology, \\ Ryukoku University, 1-5, Yokotani, Oe-cho, Seta, Otsu, Siga 520-2194, Japan \\ Fax: 81-77-543-7428, e-mail: shin@rins.ryukoku.ac.jp \\ 2) Department of Electronics and Informatics, Faculty of Science and Technology, \\ Ryukoku University, 1-5, Yokotani, Oe-cho, Seta, Otsu, Siga 520-2194, Japan \\ Fax: 81-77-543-7483, e-mail: tomizaki@rins.ryukoku.ac.jp \\ 3) Hyogo prefectural institute of technology, 3-1-12, Yukihira-cho, Suma-ku, Kobe 654-0037, Japan \\ Fax: 81-78-731-4195, e-mail: hide@hyogo-kg.go.jp \\ 4) Materials Science, NIST, 8916-5, Takayama, Ikoma, Nara, 630-0192, Japan \\ Fax: 81-743-72-6060, e-mail: uraoka@ms.naist.jp \\ 5) Panasonic Corporation, Hikaridai 3-4, Seika-cho, Kyoto 619-0237, Japan \\ Fax: 81-774-98-2517, e-mail: yamashita.ichiro@jp.panasonic.com \\ 6) Department of Physics, School of Science, University of Tokyo, 7-3-1, Hongo, Bunkyo-ku, \\ Tokyo 113-0033, Japan \\ Fax: 81-3-5841-4167, e-mail: shuji@surface.phys.s.u-tokyo.ac.jp
}

\begin{abstract}
A general atomic force microscopy (AFM) can visualize the ferritin molecule-substrate interaction directly at the single-molecule level. At present, however, it is limited by the speed at which it can successively record highly resolved images. Therefore we have studied the behavior of ferritin molecules using a high-speed atomic force microscope (high-speed AFM), which uses a miniaturized cantilever and scan stage to reduce the mechanical response time of the cantilever and to prevent the onset of resonant motion at high scan speeds. Using this instrument, we were able to achieve scan rates of up to 1 frame per second for pure fluids. In this study, we used a high-speed AFM to dynamically visualize the behavior of the ferritin molecule-substrate reaction at the single- ferritin-molecule level. We examined how long it took for the ferritin molecules to reach the substrate after they were dropped in ultrapure aqueous solution, and we also verified the effectiveness of high-speed AFM for such observation. Such direct observation is a great helpful in understanding the mechanisms by behavior of biological processes.

Key words: ferritin, high speed AFM, interaction, molecule, protein
\end{abstract}

\section{INTRODUCTION}

Nowadays, research on biotechnology is progressing rapidly. The nanometer, or the size of atoms and molecules, is now the base unit in biotechnology. In particular, the bottom-up method, in which such small atoms and molecules accumulate to form a large compound, has been enabled by the study of DNA, which has served as a "blueprint". On the basis of this blueprint, the products (amino acid residues) can be controlled at the molecular level by the control of the biological environment and the supply of elements. All the proteins that comprise various amino acid residues can form "nanoblocks" of a regular size owing to their ability to self-assemble. By integrating these nanoblocks formed by the bottom-up method involving self-assembly with conventional semiconductor fabrication technology, we have thus far proposed new process technologies for further improving the microfabrication technology for electron devices [1-4].

Following this concept, it should be possible to fabricate highly precise inorganic nanostructures using nanoblock proteins, into which inorganic substances are embedded. These proteins can be subsequently removed by an appropriate method. This new device fabrication process was developed by integrating conventional semiconductor process technology with bio-nanotechnology and is called bio-nanoprocessing [5-12].

In this study, we selected ferritin, a cage-like supermolecular protein present in the living body, for use as nanoblocks with the aim of establishing a device fabrication process using biological systems. We statically analyzed the state of two-dimensionally arranged ferritin molecules adsorbed on substrates. We also succeeded in dynamically observing the behavior of the ferritin molecules-substrate reaction in solution by real-time high-speed imaging using an AFM. Such direct observation is a great helpful in understanding the mechanisms by behavior of biological processes.

\section{Supermolecular protein, ferritin molecules}

Figure 1(a) shows the structure of ferritin. In general, ferritin in the living body has an outer shell consisting of globular protein of $13 \mathrm{~nm}$ diameter and an iron oxide core $\left(9 \mathrm{Fe}_{2} \mathrm{O}_{3} .5 \mathrm{H}_{2} \mathrm{O}\right)$ of $7 \mathrm{~nm}$ diameter [13].

The outer protein shell consists of 24 subunits, each of which consists of a polypeptide chain. These subunits 
are divided into two types, light $(\mathrm{L})$ and heavy $(\mathrm{H})$ subunits, and their proportions vary. Moreover, ferritin exhibits greater thermal and $\mathrm{pH}$ stability than most other proteins. Ferritin without the iron core is called apoferritin. Fundamentally, ferritin has a function of adjusting the amount of iron ions in the living body. That is, iron ions can be supplied and absorbed in accordance with the surrounding conditions in the presence of ferritin. To apply ferritin to the device fabrication process investigated in this study, we used Fe-filled recombinant ferritin composed of only $\mathrm{L}$ subunits, which was obtained by removing eight residues from amino acid terminals through genetic engineering.

(a)

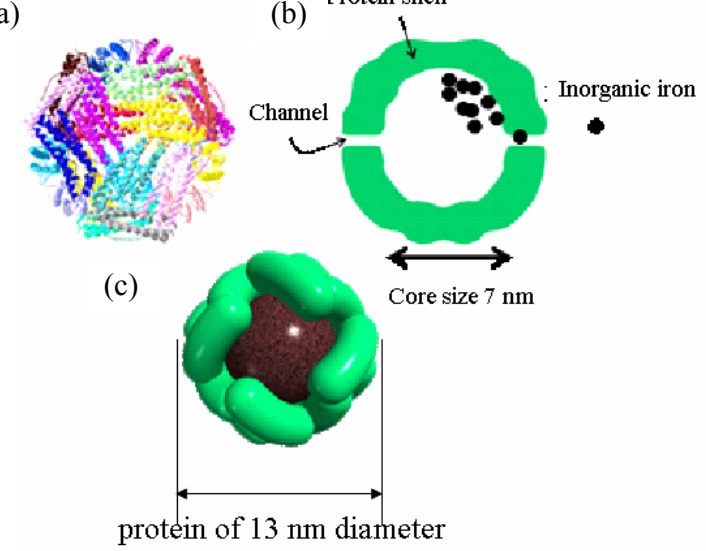

Fig. 1 (a) Appearance of ferritin, (b) Atomic model of inorganic iron in protein shell, and (c)Structure of ferritin with $\mathrm{Fe}_{2} \mathrm{O}_{3}$ core.

\section{High-Speed AFM}

AFMs enable the nanoscopic observation of the surface of specimens by scanning the surface using a probe. Unlike electron microscopes, which require a vacuum condition during observation, AFMs enable observation in solutions. It is thus possible to observe the morphology of "functioning" proteins in solutions. However, in general AFMs, it takes 5-10 minutes to obtain a frame. When a high-speed AFM is used, we can obtain 12.5 frames per second by high-speed scanning. Obtaining multiple frames per second is expected to enable new discoveries in life sciences. That is, the dynamic behavior of individual biological molecules can be nanoscopically observed without interfering with their physiological functions. Nano Live Vision (OLYMPUS, Japan) was the high-speed AFM used for the observation, which was performed in tapping mode using small probes made from silicon nitride using standard micromachining techniques [14], that were 7 $\mu \mathrm{m}$ long, $2 \mu \mathrm{m}$ wide, and $0.1 \mu \mathrm{m}$ thick. The resonance frequency of the cantilevers in water was about 636-705 $\mathrm{kHz}$ and the spring constant in water, measured by the thermal vibration method [15], was about $0.1 \mathrm{~N} / \mathrm{m}$. Each probe had an electron beam deposited probe made by Nano Tools GmbH (Munich, Germany). The 192 point $\times 144$ line images used for examining wide areas were obtained at 144 lines $/ \mathrm{sec}(1 \mathrm{sec} /$ frame $)$. The 80 point $\times$ 60 line images used for examining narrow areas were obtained at 600-900 lines/sec (0.067-0.100 sec/frame).

\section{Experimental Procedure}

4.1 Fabrication of ferritin

The inorganic cores that form inside ferritin are nanoparticles with a uniform diameter because their protein shell serves as a mold. We consider these cores of ferritin molecules as highly monodispersed nanodots. Devices fabricated using nanodots with a small variation in shape are expected to operate stably. Moreover, nanodots can be arranged at arbitrary positions by the use of the interaction between organic supermolecules and substrates. Furthermore, it is theoretically possible to fabricate two-dimensional nanodot arrays with different work functions on a single substrate by varying the type of inorganic substances embedded in the ferritin. Currently, various inorganic substances, such as $\mathrm{Fe}, \mathrm{Co}$, $\mathrm{Ni}, \mathrm{CdSe}, \mathrm{CdS}, \mathrm{ZnSe}$, and $\mathrm{InO}$, can be enclosed into ferritin through biomineralization [16].

To prepare Fe-filled ferritin molecules for use in experiments, we first purified genetically engineered recombinant apoferritin. Subsequently, a core was formed in the protein, and the protein was then purified. In addition, to confirm the conditions under which the ferritin is adsorbed onto a $\mathrm{SiO}_{2}$ substrate, we examined the change in the adsorption density of ferritin with respect to changes in the concentration of ferritin solution. Fe (II) ions in an aqueous solution were incorporated into this apoferritin via oxidation activation sites as Fe (III) ions, and Fe-fer-8 with a 7-nm-diameter $\mathrm{Fe}_{2} \mathrm{O}_{3} \cdot 5 \mathrm{H}_{2} \mathrm{O}$ core was fabricated. To avoid the generation of salt as a contaminant during the fabrication of devices, only $\mathrm{NaCl}$-free aqueous and ferritin solutions were used during the preparation of Fe-fer-8 solution.

4.2 Two-dimensional crystallization of ferritin on silicon substrate

To fabricate a two-dimensional array of Fe-filled ferritin, the ferritin is directly adsorbed onto a silicon substrate. At this stage, controlling the number of dots per unit area (dot density) is vital for obtaining good device performance. To determine the conditions under which ferritin is directly adsorbed onto a thermally grown silicon oxide film (tunnel oxide film), we examined the effects of postadesorption rinsing and the concentration of ferritin solution on the adsorption density.

In this study, for most processes we used commercially available p-Si(100) epitaxial silicon wafers, on which a 3-nm-thick thermally grown oxide film with a resistivity of $8.50-11.50 \Omega \mathrm{cm}$ was already formed. This wafer was cut into $10 \times 5 \mathrm{~mm}^{2}$ pieces and subjected to ultrasonic cleaning followed by photocleaning at $110^{\circ} \mathrm{C}$ for $10 \mathrm{~min}$ using an ultraviolet (UV) ozonizer (SAMCO, UV-1). During photocleaning, organic contaminants are degraded to volatile substances (e.g., $\mathrm{H}_{2} \mathrm{O}, \mathrm{CO}_{2}, \mathrm{~N}_{2}$, and $\mathrm{O}_{2}$ ) owing to the presence of UV light and the strong oxidation effect of $\mathrm{O}_{3}$ during its generation and degradation, and are removed from the substrate surface [17]. Furthermore, it is possible to obtain a clean $\mathrm{SiO}_{2}$ substrate surface and enhance the hydrophilic property of the surface by increasing the number of hydroxyl terminals on the substrate surface by UV ozonation. 
Next, the synthesized Fe ferritin solution was diluted using pure water to adjust the concentration to 2 or 4 $\mathrm{mg} / \mathrm{ml}$. At this time, the ferritin solution was mixed with $28 \mathrm{mM}$ MES (2-Morpholinoethanesulfonic acid monohydrate, $\mathrm{C}_{6} \mathrm{H}_{13} \mathrm{NO}_{4} \mathrm{~S} . \mathrm{H}_{2} \mathrm{O}=213.25$ ) and $27 \mathrm{mM}$ Tris (Tris [hydroxymethyl] aminomethane $\mathrm{C}_{4} \mathrm{H}_{11} \mathrm{NO}_{3}=121.1$ ) solutions to achieve a $\mathrm{pH}$ of 7.0. The reason for mixing the buffer solutions with the ferritin solution was to reduce the repulsion among ferritin molecules and increase the adsorption density.

An aliquot of the above ferritin solution was dropped onto a substrate using a pipette and allowed to adsorb on the substrate for $10 \mathrm{~min}$. Subsequently, the ferritin solution was rinsed off with ultrapure water to examine the effect of rinsing with pure water on the degree of adsorption. Next, the substrate was centrifuged at 10,000 rpm for 4 min to remove excess moisture, and then was naturally dried. To confirm the density of the adsorbed ferritin, the state of adsorption was observed using a scanning electron microscope (SEM).

4.3 Direct adsorption by centrifugal drying method

In this section, we discuss the method of directly adsorbing the ferritin onto the substrate without including any impurities such as $\mathrm{NaCl}$ or $\mathrm{MgCl}_{2}$. This direct adsorption method comprises the following steps. I. Preparing a UV-irradiated substrate

II. Dropping ferritin solution onto the substrate surface III. Removing excess ferritin solution and drying the substrate

First, we examined the difference in the amount of adsorption depending on the method of removing the excess solution and drying in step III. Here, only ferritin originating from horse spleen was used for the ferritin solution, the solvent of which was replaced with ultrapure water.

We attempted to remove the excess solution by centrifugation during the drying process. In this approach, it is important that the specimen is left in a sealed container while the excess solution is being removed. Therefore, only the excess solution was removed during centrifugation, and a single surface layer of the solution was exposed to the atmosphere immediately after the sealed container was opened to allow natural drying. This is expected to help prevent the adsorbed ferritin from forming a multilayer structure. The following is the experimental procedure used in this approach.

I. Ultrasonic cleaning of a P-type silicon substrate in acetone, methanol, and ultrapure water for $5 \mathrm{~min}$ each.

II. Removal of the substrate from the cleaning solution and then drying before dropping ferritin solution onto the substrate until its surface is covered.

III. Placing the specimen in a container and immediately sealing it to remove the excess solution by centrifugation.

IV. Removing the specimen from the sealed container and exposing it to air to allow natural drying. The rotation speed of the centrifuge is $10,000 \mathrm{rpm}$. The concentration of ferritin solution is varied from 5 to 20 $\mathrm{mg} / \mathrm{ml}$.

5.Experimental Results

5.1 Observation using high-resolution SEM (HRSEM)
We carried out SEM observation of the two-dimensional ferritin array on the silicon substrate. Figures 2(a), 2(b), and 2(c) show the observation results

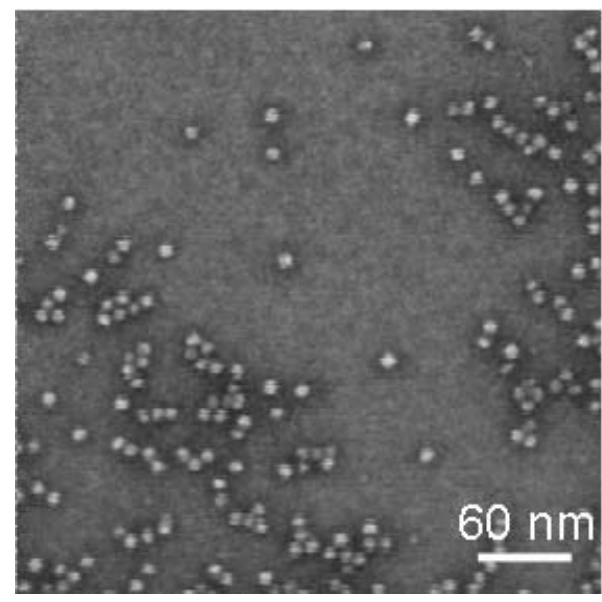

(a)

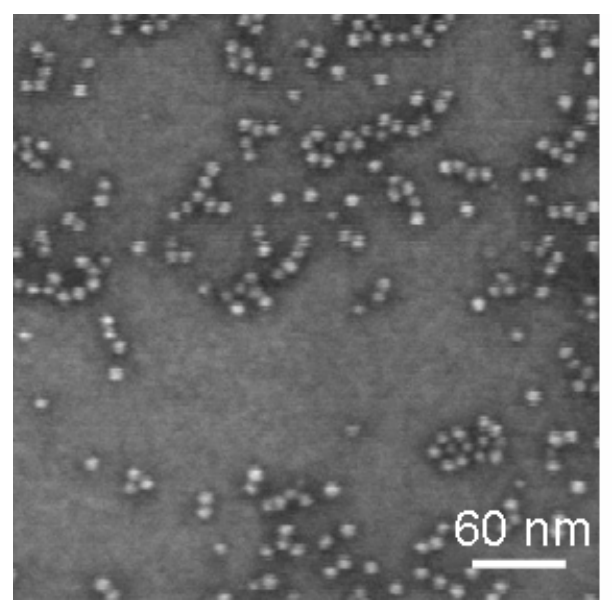

(b)

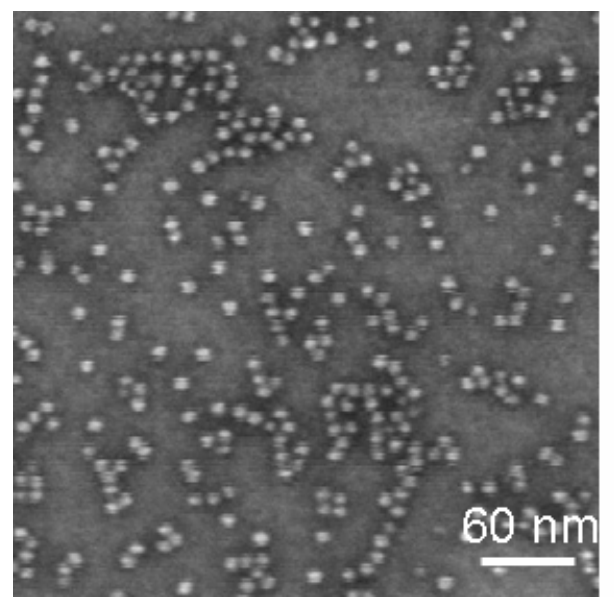

(c)

Fig. 2 Two-dimensional ferritin crystal array. (a) $5 \times 10^{13}$, (b) $1 \times 10^{14}$ and (c) $2 \times 10^{14}$ molecules $/ \mathrm{cm}^{2}$. 
for dot densities of $5 \times 10^{13}, 1 \times 10^{14}$, and $2 \times 10^{14}$ molecules $/ \mathrm{cm}^{2}$, respectively; white globular ferritin cores were observed. No multilayer regions of ferritin due to drying were observed, and the formation of a single-layer array was confirmed over almost the entire region. Next, we evaluated the difference in the amounts of ferritin adsorbed between hydrophilic and hydrophobic substrate surfaces. The hydrophilic surface was obtained by irradiating the natural oxide film on the surface of the silicon substrate with UV light. Subsequently, the substrate was carefully rinsed with ultrapure water. Here, the concentration of the ferritin solution was $20 \mathrm{mg} / \mathrm{ml}$, and pure water was used as its solvent.

Figure 3 shows the ferritin adsorbed on the hydrophilic and hydrophobic surfaces. The conditions of the surfaces are (a) hydrophilic, (b) slightly hydrophilic, and (c) slightly hydrophobic. It was confirmed that the amount of ferritin adsorbed on the hydrophobic surface was much smaller than that on the hydrophilic surface. This may be because the amount of solution remaining on the substrate surface, which depends on the surface tension and the hydrophilic or hydrophobic property, differs between these surfaces.

\subsection{Observation using high-speed AFM}

By carrying out the AFM observation of ferritin molecules, we examined how long it took for the ferritin molecules to reach the substrate after they were dropped in ultrapure aqueous solution, and we also verified the effectiveness of high-speed AFM for such observation. Figure 4 shows high-speed AFM images of the ferritin molecules, which were obtained by continuous imaging (1 s/frame). As shown in Fig. 4(a), it was confirmed by the AFM observation of ferritin molecules in ultrapure aqueous solution that no ferritin molecules were initially present over a $170 \times 230 \mathrm{~nm}^{2}$ region (first image). The images in Fig. 4 were obtained by scanning the specimen from right to left in the $\mathrm{X}$ direction. Next, the ferritin solution was dropped to give a dot density of $1 \times$ $10^{13}$ molecules $/ \mathrm{cm}^{2}$. Ferritin molecules are not observed in Fig. 4(a), but were clearly observed $15 \mathrm{~s}$ after the start of the measurement (16th image), as shown in Fig. 4(b). The high-speed AFM enabled us to directly visualize the dynamic behavior of a two-dimensional array of "functioning" protein molecules as real images, which could previously be predicted from data indirectly obtained. Between the images taken 191 s (Fig. 4(c)) and $278 \mathrm{sec}$ (Fig. 4(d)) after the start of the measurement, an adsorbed ferritin molecule is dissociated from the substrate by the physical force applied by a scanning probe (indicated by '*' in Figs. 4(c) and 4(d) or in Figs. 4(i) and 4(j)). This may be due to the weak adsorption between the ferritin molecule and the substrate in the aqueous solution. After $600 \mathrm{sec}$ from the start of the measurement (Fig. 4(g)), a third ferritin molecule was observed. Moreover, after the fourth ferritin molecule was adsorbed onto the substrate after $990 \mathrm{sec}$ (Fig. 4(k)), no more ferritin molecules were adsorbed. After the high-speed AFM images up to $1485 \mathrm{sec}$ (Fig. 4(p)) were obtained, the scanning was stopped. When the cross-sectional profile of the ferritin protein, indicated by the arrow in Fig. 4(b), was subsequently obtained in the solution, the height of the ferritin protein was maintained at $13 \mathrm{~nm}$, as shown in Fig. 5. This indicates that the ferritin molecules were not significantly

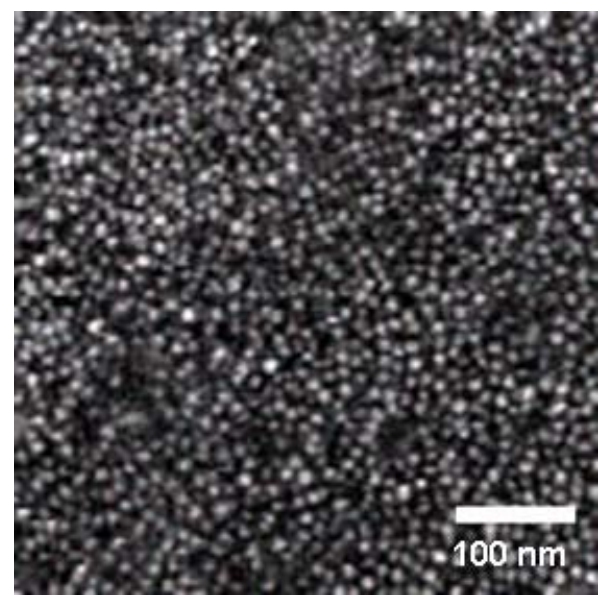

(a)

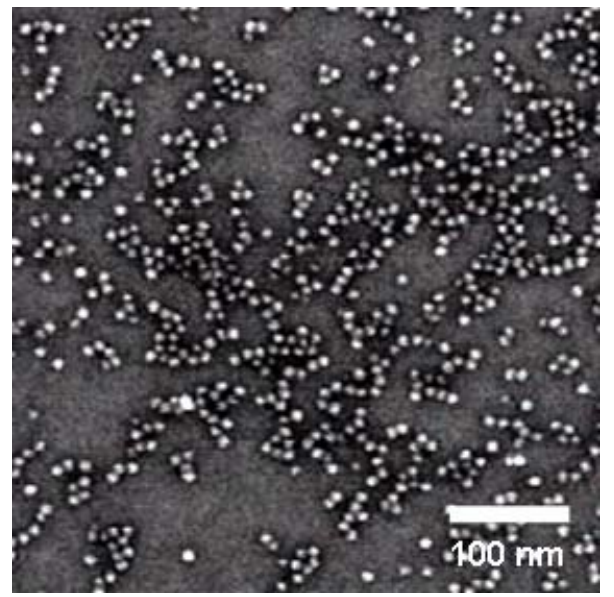

(b)

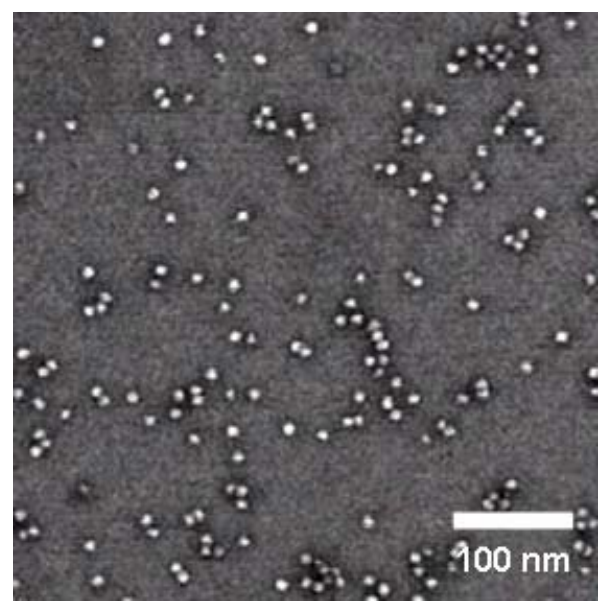

(c)

Fig. 3 Difference in amounts of ferritin adsorbed between hydrophilic and hydrophobic substrate surfaces. (a) Hydrophilic, (b) slightly hydrophilic, and (c) slightly hydrophobic areas. 


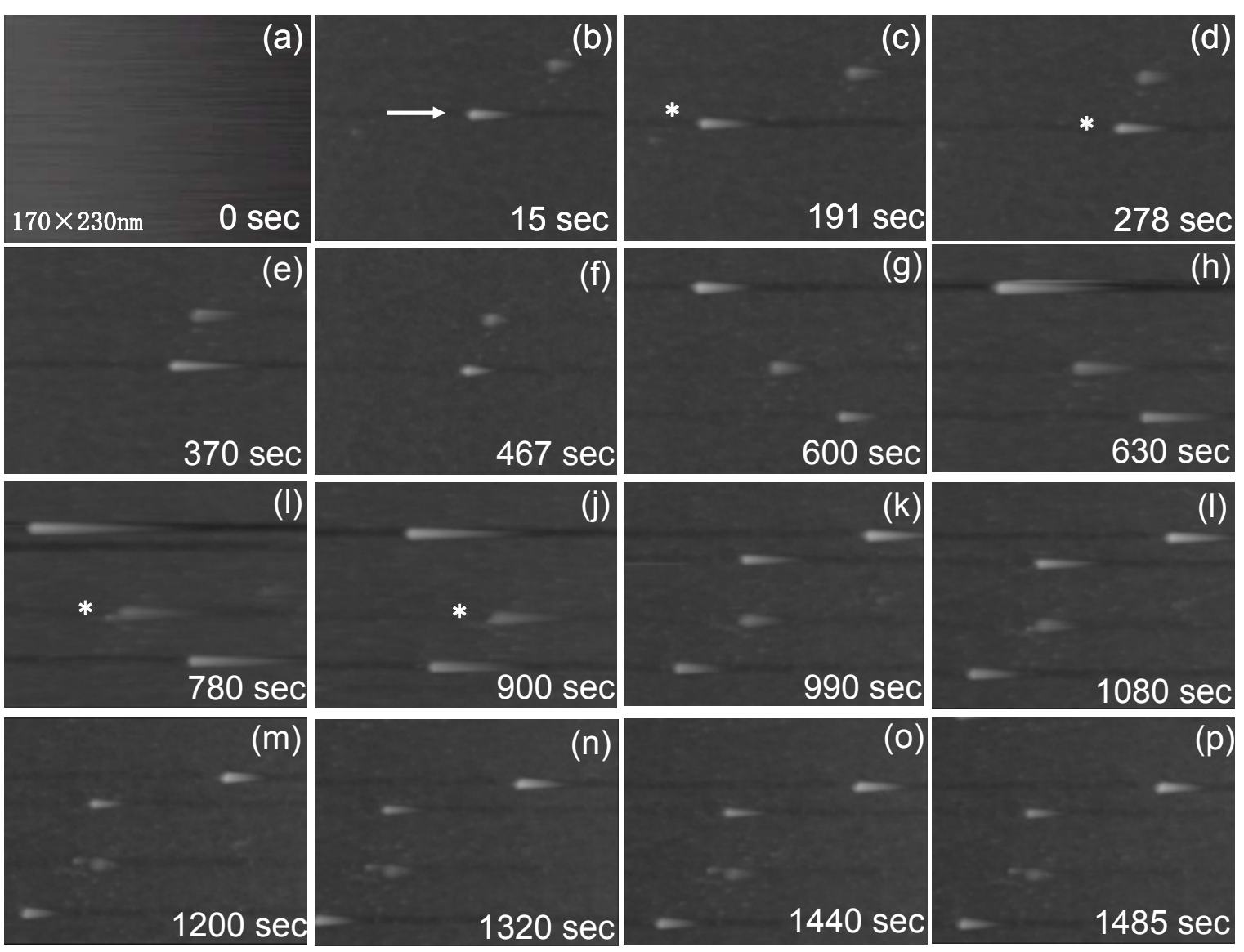

Fig. 4 Dynamic images of ferritin molecule-substrate interaction in pure water. (a) First image before adding ferritin solution. (b-p) Dynamic images of ferritin molecules after adding ferritin solution. Numbers at the bottom right in each image indicate time to reach the substrate after dropping the ferritin molecules in seconds.

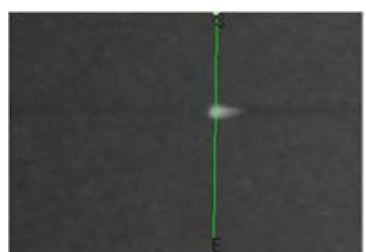

(a)

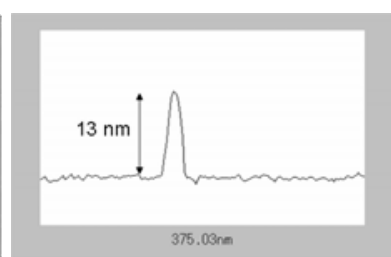

(b)
Fig. 5 Observation of ferritin protein molecule in pure water. (a) AFM image and (b) cross-sectional profile of (a).

damaged even after continuous imaging by the high-speed AFM. As the shape of the ferritin was clearly captured, we succeeded in accurately measuring the surface shape, which indicates that high-speed AFM is even effective for the measurement of brittle specimens, such as ferritin molecules. Therefore, high-speed AFMs are very powerful tools for measuring brittle protein molecules, which is difficult using conventional equipment.

\section{Conclusions}

Owing to the improved scanning speed of AFM imaging, we succeeded in the real-time observation of the process by which ferritin molecules are adsorbed onto substrates. High-speed AFM imaging even enables the observation of ferritin molecules that are not fully immobilized on substrates and are moving freely in a solution. In addition to successfully observing the adsorption process of ferritin on a substrate in solution, we also observed the dissociation of a ferritin molecule from the substrate by the physical force applied by the probe during imaging. High-speed AFM imaging is an effective method of analysis that can trace the dynamic behavior of biological molecules, such as their responses to external stimuli and chemical reactions, in real time.

\section{Acknowledgment}

We thank Dr. Takao Okada at the Research Institute of Biomolecule Metrology Co., Ltd., for his valuable advice regarding the high-speed AFM. This work was supported by the Joint Research Center for Science and Technology of Ryukoku University.

\section{References}

[1] S-I. Yamamoto, H. Yoshioka, Y.Uraoka, T. Fuyuki, M. Okuda and I. Yamashita: Jpn. J. Appl. Phys. vol. 46, No. 8B (2007) 5647. 
[2] S-I. Yamamoto, H. Yoshioka, Y. Uraoka, T. Fuyuki, and I. Yamashita: Journal of Physics: 61 (2007) 1276.

[3] S-I. Yamamoto, K. Kobayashi, H. Yamada, H. Yoshioka, Y. Uraoka, T. Fuyuki, and I. Yamashita: Journal of Physics: 100 (2008) 052004.

[4] T. Hikono, Y. Uraoka, T. Fuyuki I. Yamashita: Jpn. J. Appl. Phys. Vol.42 (2003) L.398.

[5] S-I. Yamamoto, H. Yoshioka, Y.Uraoka, T. Fuyuki, M. Okuda and I. Yamashita: Jpn. J. Appl. Phys. vol. 47, No. 7 (2008) 6160

[6] I. Yamashita, Thin Solid Films, 393 (2001) 12.

[7] T. Hikono, Y. Uraoka, T. Fuyuki, and I. Yamashita, Jpn. J. Appl. Phys. 42 (2003) 398.

[8] T. Hikono, A. miura, T. Matsumura, Y. Uraoka, T. Fuyuki, S. Yoshii, I. Yamashita and M. Takeguchi, Appl. Phys. Lett. 88 (2006) 023108.

[9] A. Miura, T. Hikono, T. Matsumura, H. Yano, T. Hatayama, Y. Uraoka, T. Fuyuki, S. Yoshii and I. Yamashita, Jpn. J. Appl. Phys. 45 (2006) n1. L1.

[10] T. Furuno, H. Sasabe and K. Ulmer, Thin Solid Films, 180 (1989) 23.

[11] A. Sato, T. Furuno, C. Toyoshima and H. Sasabe, Biochem. Biophys. Acta.,1162 (1993) 54.

[12] E. Adachi and K. Nagayama, Langmuir, 12 (1996) 1836.

[13] W. H. Massover, Micron. 24(4) (1993) 389.

[14] M. Kitazawa, K. Shiotani, A. Toda. 2003. Batch fabrication of sharpened silicon nitride tips. Jpn. J. Appl. Phys. 42:4844.

[15] J. L. Hutter, J. Bechhoefer. 1993. Calibration of atomic-force microscope tips. Rev. Sci. Instrum. 64 (2000) 1868.

[16] H. Kirimura, Y. Uraoka, T. Fuyuki, M. Okuda, and I. Yamashita, Appl. Phys. Lett. 86 (2005) 262106.

[17] John R. Vig, J. Vac. Sci. Technol. A 3(3) (1985) 1027. 